\title{
L'exploration et le traitement de la région axillaire des tumeurs infiltrantes du sein : parties I à IV - Tableau synthétique (RPC 2013)*
}

Recommandations sur l'exploration de l'aisselle

Niveau

de preuve Grade

Prise en charge du (des) ganglion(s) sentinelle(s) en techniques conventionnelles

L'analyse extemporanée du ganglion sentinelle ne se justifie que si l'on envisage une totalisation du curage axillaire en cas de positivité du (des) ganglion(s) sentinelle(s)

Accord d'experts

En cas d'examen extemporané, il n'existe pas d'arguments clairs permettant de préférer une des techniques de routine (coupe congelée ou cytologie)

2

Les recommandations sur la meilleure méthode d'analyse du ganglion sentinelle sont soumises à la signification pronostique et à l'attitude thérapeutique face aux atteintes minimes (micrométastases et cellules tumorales isolées). Si l'on veut seulement diagnostiquer les micrométastases, la plupart des études considèrent que des coupes sériées espacées de $250 \mu \mathrm{m}$ au plus suffisent et que l'immunohistochimie ne se justifie pas. La recherche des cellules tumorales isolées nécessitera par contre le recours à l'immunohistochimie

Prise en charge locorégionale complémentaire du (des) ganglion(s) sentinelle(s) micrométastatique(s)

L'abstention chirurgicale axillaire en cas de ganglion(s) sentinelle(s) micrométastatique(s) après validation en RCP préthérapeutique peut être une option

1

Si un traitement conservateur du sein avec une radiothérapie du sein est planifié

Si un traitement adjuvant (chimiothérapie et/ou traitement antihormonal) est prévu sur des critères autres que l'absence de curage axillaire complémentaire

L'abstention de curage complémentaire en cas de ganglion(s) sentinelle(s) micrométastatique(s) ne doit pas être compensée par une augmentation dans la prescription de chimiothérapie

Accord d'experts

Prise en charge locorégionale complémentaire du (des) ganglion(s) sentinelle(s) macrométastatique(s)

En cas de macrométastase (du/des) ganglion(s) sentinelle(s), un curage axillaire complémentaire est indiqué

En cas de mastectomie et/ou de la présence de plus de deux ganglions sentinelles macrométastatiques, aucune donnée n'est disponible pour envisager l'abstention du curage axillaire

La morbidité liée au curage axillaire après ganglion sentinelle apparaît équivalente quel que soit le moment du curage

Le taux attendu de ganglions non sentinelles envahis au curage axillaire complémentaire peut être évalué pour chaque patiente par des modèles (nomogrammes ou scores). Ces outils de prédiction du risque d'envahissement des ganglions non sentinelles peuvent être utilisés en pratique clinique pour discuter de la réalisation ou non d'un curage axillaire complémentaire en fonction du choix des patientes ou de leur refus du curage et dans des cas particuliers (âge, comorbidités importantes...) où le bénéfice du curage est discutable

Place de l'exploration du (des) ganglion(s) sentinelle(s) macrométastatique(s) en cas de chimiothérapie néoadjuvante Avant chimiothérapie néoadjuvante, en cas de tumeur opérable d'emblée, si le ganglion sentinelle n'est pas détecté ou métastatique, un curage axillaire doit être réalisé, avant ou après la chimiothérapie néoadjuvante La technique de la double détection du ganglion sentinelle avant puis après la chimiothérapie néoadjuvante est à proscrire

$\begin{array}{ll}1 & \text { A } \\ 1 & \text { A } \\ 1 & \text { A } \\ & \\ 2 & \text { B }\end{array}$

Les patientes $\mathrm{N}+$ avant le traitement néoadjuvant dont l'aisselle aura été " traitée " par ce même traitement systémique devront avoir un curage axillaire. La technique du ganglion sentinelle n'est pas recommandée La technique du ganglion sentinelle peut être proposée, après validation en RCP préthérapeutique et information claire et loyale de la patiente, à condition qu'au moins deux ganglions sentinelles soient prélevés et qu'une évaluation échographique axillaire ( \pm cytoponction) avant traitement ait été réalisée ne montrant pas de ganglion suspect

Les experts recommandent de poursuivre les efforts d'inclusion des patients dans les essais qui comparent le curage axillaire avec l'abstention en cas de ganglion sentinelle métastatique ainsi que la validation de la stratégie d'exploration axillaire optimale en cas de traitements néoadjuvants

\footnotetext{
* Ce tableau se réfère aux quatre parties de l'article « L'exploration et le traitement de la région axillaire des tumeurs infiltrantes du sein (RPC 2013) » (DOI 10.1007/s10269-013-2337-z, DOI 10.1007/s10269-013-2338-y, DOI 10.1007/s10269-013-2339-x, DOI 10.1007/s10269-013-2340-4).
} 